\title{
Rapid diagnosis of pulmonary tuberculosis by combined molecular and immunological methods
}

\author{
Claudia Jafari ${ }^{1,6}$, loana D. Olaru $\mathbb{0}^{1,2,6}$, Franziska Daduna ${ }^{1}$, Martin Ernst ${ }^{3}$, \\ Jan Heyckendorf $\mathbb{1}^{1,4}$, Christoph Lange ${ }^{1,4,5}$ and Barbara Kalsdorf ${ }^{1,4}$
}

Affiliations: ${ }^{1}$ Division of Clinical Infectious Diseases, Research Center Borstel, Borstel, Germany. ${ }^{2}$ Biomedical Research and Training Institute, Harare, Zimbabwe. ${ }^{3}$ Division of Immune Cell Analytics, Research Center Borstel, Borstel, Germany. ${ }^{4}$ International Health/Infectious Diseases, University of Lübeck, German Center for Infection Research (DZIF), Partner Site Hamburg-Lübeck-Borstel, Lübeck, Germany. ${ }^{5}$ Dept of Medicine, Karolinska Institutet, Stockholm, Sweden. ${ }^{6}$ Both authors contributed equally.

Correspondence: Christoph Lange, Medical Clinic, Research Center Borstel, Parkallee 35, 23845 Borstel, Germany. E-mail: clangeafz-borstel.de

@ERSpublications

Nearly all patients with active pulmonary TB can be rapidly identified when two diagnostic methods are combined http://ow.ly/vinu30j5Yk7

Cite this article as: Jafari C, Olaru ID, Daduna F, et al. Rapid diagnosis of pulmonary tuberculosis by combined molecular and immunological methods. Eur Respir J 2018; 51: 1702189 [https://doi.org/10.1183/ 13993003.02189-2017].

ABSTRACT Diagnosing pulmonary tuberculosis (TB) may be delayed until culture results become available.

We ascertained the accuracy of a stepwise diagnostic algorithm for the rapid diagnosis of pulmonary TB by GeneXpert from sputum and/or bronchoalveolar lavage (BAL) followed by a Mycobacterium tuberculosis-specific BAL ELISPOT assay in patients with a suspected diagnosis of pulmonary TB at a clinical referral centre in Germany.

Among 166 patients with a presumptive diagnosis of pulmonary TB, 81 cases were confirmed by M. tuberculosis culture from sputum and/or BAL. In 66 out of 81 (81.5\%) cases, patients initially had M. tuberculosis detected by GeneXpert from sputum; in addition, six out of $81(7.4 \%)$ cases were diagnosed by GeneXpert on BAL fluid (together 72 out of 81 (88.9\%) patients). Out of the remaining nine patients with negative GeneXpert results from sputum and BAL, BAL ELISPOT identified eight patients with culture-confirmed TB correctly (median time to culture positivity 26 days). At a cut-off of $>4000$ early secretory antigenic target-6- or culture filtrate protein-10-specific interferon- $\gamma$-producing lymphocytes per 10000000 lymphocytes, the specificity of the BAL ELISPOT for active TB was 97\%.

In low $\mathrm{TB}$ incidence countries, nearly all patients with active pulmonary $\mathrm{TB}$ can be identified within the first few days of clinical presentation using a stepwise strategy with GeneXpert and BAL ELISPOT. 


\section{Introduction}

According to the World Health Organization, 10.4 million new cases of tuberculosis (TB) occurred worldwide in 2016 [1]. Early diagnosis of pulmonary TB is crucial to avoid transmission of Mycobacterium tuberculosis. M. tuberculosis culture remains the gold standard for TB diagnosis, but it takes $2-5$ weeks on average until culture results become available $[2,3]$. The detection of acid-fast bacilli (AFB) by sputum smear microscopy is a widely used, inexpensive screening test in patients with a presumptive diagnosis of pulmonary TB. However, $<50 \%$ of all notified cases are sputum smear-positive [4]. Furthermore, microscopy does not distinguish M. tuberculosis from other mycobacterial species. Nucleic acid amplification techniques such as GeneXpert (Xpert MTB/RIF; Cepheid, Sunnyvale, CA, USA) have a short time to result of $<2 \mathrm{~h}$, and are highly sensitive and specific for the detection of $M$. tuberculosis DNA on AFB smear-positive samples. However, the sensitivity of GeneXpert for the diagnosis of pulmonary TB decreases to $<70 \%$ for smear-negative cases [5]. Thus, $\sim 15 \%$ of adult patients with pulmonary TB remain undetected when initially investigated by GeneXpert.

Following contact with antigen-presenting cells, M. tuberculosis-specific T-cells clonally expand in regional lymph nodes and migrate to the site of the infection where they differentiate into effector T-cells [6-8]. After loss of special phenotypical T-cell surface markers, these cell populations are probably unable to re-enter the bloodstream and become enriched at the site of disease $[9,10]$. In contrast to detection of M. tuberculosis-specific effector T-cells in peripheral blood, detection of M. tuberculosis-specific effector T-cells by the interferon (IFN)- $\gamma$ release assay at the site of the infection has a high diagnostic accuracy for active TB even when AFB and $M$. tuberculosis DNA are not detectable from local samples such as bronchoalveolar lavage (BAL) [11-15], pleural fluid [16, 17], pericardial fluid [18], cerebrospinal fluid [19] or ascites [20]. ELISPOT test results are available within $24 \mathrm{~h}$.

In order to ascertain the diagnostic accuracy of the stepwise testing of sputum or BAL by GeneXpert followed by $M$. tuberculosis-specific BAL ELISPOT for the rapid diagnosis of active pulmonary TB, we prospectively enrolled patients with a presumptive diagnosis of pulmonary $\mathrm{TB}$ at a clinical $\mathrm{TB}$ referral centre in Germany.

\section{Methods}

\section{Study participants}

We performed a prospective analysis of all patients (age $\geqslant 17$ years) admitted to the Medical Clinic of the Research Center Borstel, Germany, between November 2011 and June 2016, presenting with symptoms and/or chest radiography suggestive of pulmonary TB. Patients who had received treatment for TB within the 2 years prior to admission and patients with extrapulmonary TB were excluded from the study. As the initial diagnosis, sputum smear microscopy and/or GeneXpert was performed on up to three sputum samples. In the absence of detectable AFB in smears and/or negative M. tuberculosis-specific nucleic acid tests on sputum, bronchoscopy with BAL was performed according to national guidelines [21]. Pooled BAL was divided into two aliquots: one for ELISPOT, and one that was sent for BAL microscopy, GeneXpert and culture to the National Reference Center for Mycobacteria in Borstel. The study flowchart is shown in figure 1.

The study was approved by the Ethics Committee of the University of Lübeck (14-031A). Reporting follows the STARD criteria [22].

GeneXpert

GeneXpert was performed on sputum and BAL according to the manufacturer's guidelines at the National Reference Center for Mycobacteria [23].

\section{M. tuberculosis-specific ELISPOT}

M. tuberculosis-specific ELISPOT was performed on peripheral blood mononuclear cells (PBMCs) and BAL cells (BALCs) as previously described [12]. Results of $M$. tuberculosis-specific ELISPOT were considered positive if more than five spot-forming cells (SFCs) were observed in the early secretory antigenic target (ESAT)-6 or the culture filtrate protein (CFP)-10 well, after subtracting the number of SFCs in the negative control well, and if the total number of SFCs in the ESAT-6 or CFP-10 well was at least twice the number of SFCs in the negative control well. The results were considered negative if they did not meet the definition for a positive result and if the number of SFCs in the positive control (anti-CD3, clone X35, $10 \mathrm{ng} \cdot \mathrm{mL}^{-1}$; Beckman Coulter, Krefeld, Germany) was $>20$ SFCs after subtracting the number of SFCs in the negative control well and if the positive control had at least twice the number of SFCs of the negative control well. Indeterminate results were defined as not meeting the criteria for a positive or a negative test result. Due to the limited number of cells in BAL, some ELISPOT tests were performed with less than 250000 cells per well; these results were normalised for 250000 cells per well. 


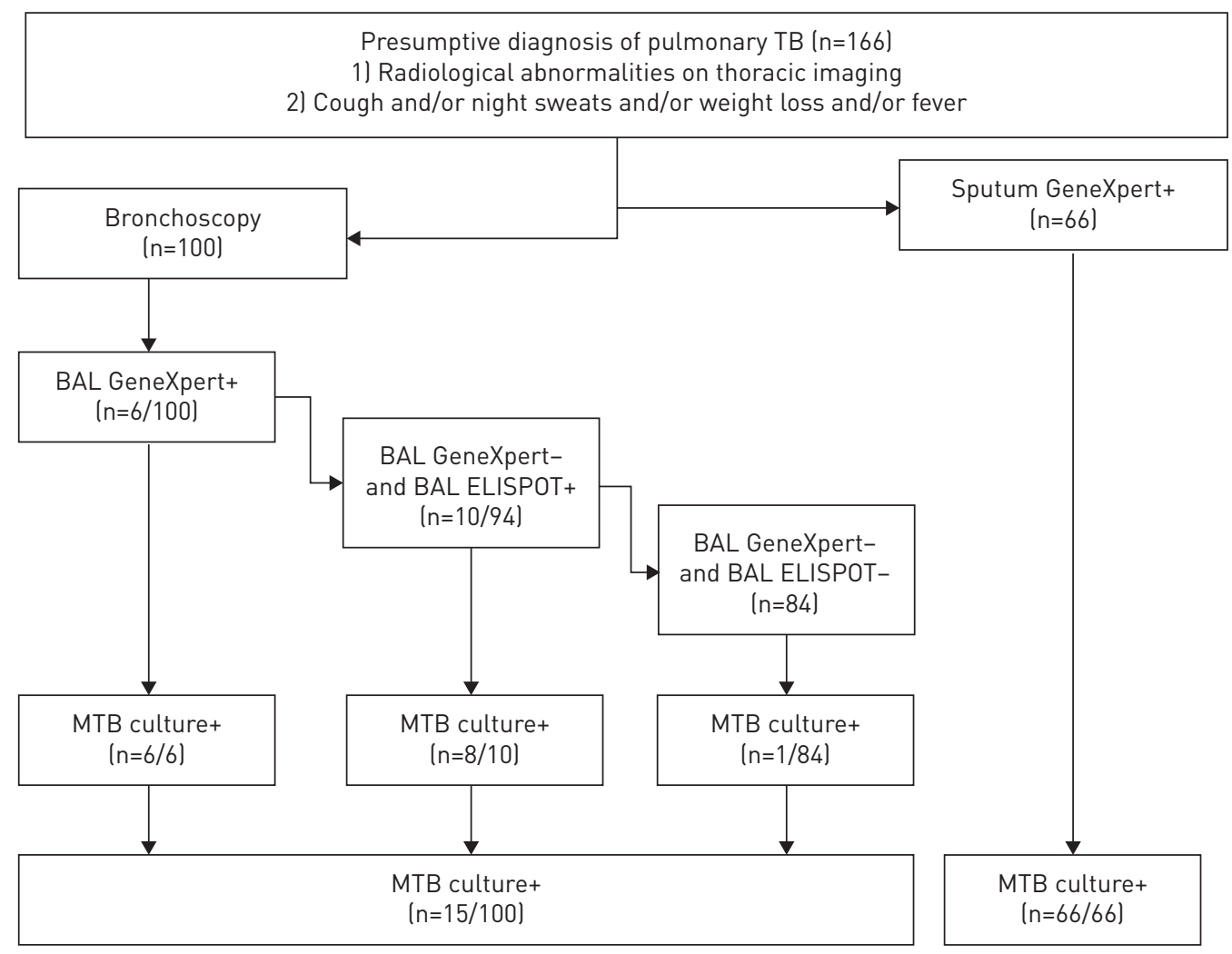

FIGURE 1 Study flowchart. TB: tuberculosis; BAL: bronchoalveolar lavage; MTB: Mycobacterium tuberculosis.

Defining the lymphocyte population by flow cytometry analysis

To adjust for the different percentages of lymphocytes in blood and lavage, 500000 PBMCs or BALCs were acquired on a FACSCalibur flow cytometer (BD Bioscience, Heidelberg, Germany). The respective percentage of lymphocytes out of the population of PBMCs or BALCs was used to extrapolate SFCs per 250000 cells per well to 1000000 lymphocytes in blood or BAL specimens [12]. Recruitment of M. tuberculosis-specific lymphocytes to the lungs was calculated by dividing the number of SFCs per 1000000 lymphocytes in BALCs by the number of SFCs per 1000000 lymphocytes in peripheral blood. As previously described, if the denominator had zero SFCs, a value of 0.1 was assigned $[12,24]$.

\section{Statistical analysis}

Statistical analysis was performed using Stata version 14 (StataCorp, College Station, TX, USA) and CATmaker version 1.1 (Center for Evidence-Based Medicine, Oxford, UK). The Mann-Whitney U-test was used to compare groups of continuous variables. The cut-offs were selected using receiver operating characteristic (ROC) curve analysis. The significance level was set at $\alpha=0.05$.

\section{Results}

Between November 1, 2011 and June 30, 2016, 412 patients with a presumptive or confirmed diagnosis of TB were admitted to the Medical Clinic of the Research Center Borstel. Of these, 196 patients already had a confirmed diagnosis of pulmonary TB. Another 50 patients were diagnosed with extrapulmonary TB. These patients were excluded. Of the remaining 166 individuals, who were admitted with presumptive pulmonary TB, 66 patients had a microbiological confirmation of the initial diagnosis made by a positive GeneXpert result on sputum. The remaining 100 individuals, in whom the diagnosis of TB could not be confirmed by sputum smear microscopy and sputum GeneXpert, but where pulmonary TB was still part of the differential diagnosis, underwent bronchoscopy with BAL.

The median (interquartile range (IQR)) age of these 100 patients was $52.0(35.5-63.0)$ years and 61 (61\%) were male.

One patient with culture-confirmed TB and one patient without TB were tested HIV-positive. 
15 out of 100 patients had culture-confirmed TB. Six patients with culture-confirmed TB (40\%) had a rapid diagnosis of TB by a positive BAL GeneXpert result (of whom two also had detectable AFB on BAL microscopy; four did not have detectable AFB in BAL). Thus, there were nine out of 100 (9\%) patients with negative test results on sputum and BAL by microscopy and GeneXpert that had culture-confirmed diagnosis of $\mathrm{TB}$ with a median (IQR) time to culture positivity of 26 (16.5-34.5) days. Patient characteristics are shown in table 1.

Among the 100 patients who underwent bronchoscopy, four were diagnosed with nontuberculous mycobacterial (NTM) pulmonary infections. Two patients had evidence of AFB in BAL and GeneXpert did not detect $M$. tuberculosis; the mycobacterial species identified from culture in these patients were Mycobacterium avium and Mycobacterium simiae. Two individuals with negative results on BAL microscopy and GeneXpert had a positive culture from BAL for Mycobacterium xenopi and Mycobacterium kansasii. Samples from the remaining 81 patients did not show mycobacterial growth. Patient characteristics and details on the final diagnosis of the patients who underwent bronchoscopy but were ultimately diagnosed as not having pulmonary TB are shown in supplementary table S1.

\section{PBMC and BALC ELISPOT}

Among 100 patients with a presumptive diagnosis of pulmonary $\mathrm{TB}$, one had an indeterminate test result in PBMCs. In one out of 100 (1\%) there was failure of the positive control in the PBMC ELISPOT. In five out of $100(5 \%)$ the BALC ELISPOT was indeterminate: two had failure of the negative control and three had failure of the positive control. Using the manufacturer's definition of a positive test result, among the patients with valid test results, PBMC and BALC ELISPOT were positive in 62 out of $99(62.6 \%)$ and 40 out of 95 (42.1\%) individuals, respectively. Active TB could be excluded in 34 out of 62 (54.8\%) individuals with a positive PBMC ELISPOT and 25 out of 40 (62.5\%) individuals with a positive BALC ELISPOT. Out of 15 patients with culture-confirmed pulmonary TB, PBMC ELISPOT was positive in 14 out of $15(93.3 \%)$ and negative in one out of 15 (6.7\%). BALC ELISPOT was positive in 15 out of $15(100 \%)$.

For patients with culture-confirmed pulmonary TB, PBMC ELISPOT had a sensitivity of 93\% (95\% CI $81-100 \%)$ and a specificity of 50\% (95\% CI 38-62\%), while BALC ELISPOT had a sensitivity of $100 \%$ and a specificity of $77 \%$ (95\% CI $67-87 \%)$ when the manufacturer's definitions of positive and negative test results on PBMCs were used.

Table 2 shows all individual test results for the nine culture-confirmed pulmonary TB patients with negative AFB and negative GeneXpert results in BAL. The respective numbers of positive or negative paired PBMC and BALC ELISPOT results in patients with culture-confirmed pulmonary TB and patients without TB are given in supplementary figure $\mathrm{S} 1$.

\section{Concentration of antigen-specific SFCs at the site of infection}

In patients with culture-confirmed pulmonary TB, the median (IQR) SFC count was 25 (6.3-210) per 250000 PBMCs for ESAT-6 and 53.8 (22.5-177) per 250000 PBMCs for CFP-10. BALC ELISPOT median (IQR) was elevated to 295 (52.5-489) SFCs per 250000 BALCs for ESAT-6 and 160 (61-453) SFCs per

TABLE 1 Individual test results and patient characteristics of the nine patients with culture-confirmed tuberculosis with undetectable acid-fast bacilli (AFB) in bronchoalveolar lavage (BAL) and negative GeneXpert results

\begin{tabular}{|c|c|c|c|c|c|c|c|c|c|}
\hline Patient & $\begin{array}{l}\text { Age } \\
\text { years }\end{array}$ & Sex & $\begin{array}{c}\text { Country of } \\
\text { origin }\end{array}$ & $\begin{array}{c}\text { MTB } \\
\text { culture }\end{array}$ & $\begin{array}{l}\text { AFB } \\
\text { BAL }\end{array}$ & $\begin{array}{c}\text { GeneXpert } \\
\text { BAL }\end{array}$ & $\begin{array}{c}\text { PBMC } \\
\text { ELISPOT }\end{array}$ & $\begin{array}{c}\text { BALC } \\
\text { ELISPOT }\end{array}$ & $\begin{array}{l}\text { TPP } \\
\text { days }\end{array}$ \\
\hline 1 & 30 & Male & Somalia & + & - & - & + & + & $17^{\#}$ \\
\hline 2 & 31 & Male & Ghana & + & - & - & + & + & $31^{\#}$ \\
\hline 3 & 69 & Male & Germany & + & - & ITR & + & + & $16^{\text {ๆ }}$ \\
\hline 4 & 62 & Female & Afghanistan & + & - & - & + & + & $38^{\text {श }}$ \\
\hline 5 & 27 & Female & Germany & + & - & - & + & + & $21^{\text {ๆ }}$ \\
\hline 6 & 43 & Male & Germany & + & - & - & + & + & $16^{\#}$ \\
\hline 7 & 19 & Female & Germany & + & - & - & + & + & $28^{9}$ \\
\hline 8 & 48 & Male & Russia & + & - & - & + & + & $26^{\text {व }}$ \\
\hline 9 & 33 & Male & Eritrea & + & - & - & + & + & $39^{\#}$ \\
\hline
\end{tabular}

MTB: Mycobacterium tuberculosis; PBMC: peripheral blood mononuclear cell; BALC: BAL cell; TPP: time to culture positivity of the first culture growing MTB; ITR: indeterminate test result. ${ }^{\#}$ : sputum; " ${ }^{\text {; }}$ BAL. 
TABLE 2 Individual ELISPOT results and recruitment factors for the nine patients with culture-confirmed tuberculosis with negative acid-fast bacilli microscopy and negative GeneXpert results in bronchoalveolar lavage (BAL)

\begin{tabular}{|c|c|c|c|c|c|c|c|c|c|c|}
\hline \multirow[t]{3}{*}{ Patient $^{\#}$} & \multicolumn{4}{|c|}{ SFCs per 250000 cells } & \multicolumn{4}{|c|}{ SFCs per 1000000 lymphocytes } & \multicolumn{2}{|c|}{$\begin{array}{l}\text { Recruitment } \\
\text { factor }\end{array}$} \\
\hline & \multicolumn{2}{|c|}{ PBMCs } & \multicolumn{2}{|c|}{ BALCs } & \multicolumn{2}{|c|}{ PBMCs } & \multicolumn{2}{|c|}{ BALCs } & \multirow[t]{2}{*}{ ESAT-6 } & \multirow[t]{2}{*}{ CFP-10 } \\
\hline & ESAT-6 & CFP-10 & ESAT-6 & CFP-10 & ESAT-6 & CFP-10 & ESAT-6 & CFP-10 & & \\
\hline 1 & 473 & 301 & 407 & 279 & 3300 & 2100 & 15654 & 10731 & 4.7 & 5.1 \\
\hline 2 & 210 & 195 & 526 & 351 & 1761 & 1635 & 6370 & 4251 & 3.6 & 2.6 \\
\hline 3 & 291 & 64 & 450 & 621 & 1229 & 270 & 4780 & 6599 & 3.9 & 24.4 \\
\hline 4 & 6 & 6 & 546 & 479 & 44 & 44 & 12248 & 10734 & 279.2 & 244.7 \\
\hline 5 & 2.5 & 10 & 251 & 160 & 12 & 47 & 6058 & 3858 & 514.9 & 81.9 \\
\hline 6 & 25 & 23 & 295 & 349 & 122 & 110 & 18820 & 21930 & 145.3 & 199.8 \\
\hline 7 & 15 & 33 & 176 & 159 & 73 & 161 & 5949 & 5359 & 81.3 & 33.3 \\
\hline 8 & 20 & 29 & 52.5 & 50 & 113 & 162 & 4595 & 4376 & 40.8 & 27 \\
\hline 9 & 5 & 47 & 6 & 61 & 24 & 229 & 107 & 1085 & 4.4 & 4.7 \\
\hline
\end{tabular}

SFC: spot-forming cell; PBMC: peripheral blood mononuclear cell; BALC: BAL cell; ESAT: early secretory antigenic target; CFP: culture filtrate protein. "\#: see table 1 for patient characteristics; ": SFCs in BALCs/ SFCs in PBMCs multiplied by the lymphocyte factor $1 \%$ of lymphocytes in PBMCs $/ \%$ lymphocytes in BALCs).

250000 BALCs for CFP-10. In contrast, patients without TB had a median (IQR) of 3.8 (0.1-17.5) SFCs per 250000 PBMCs for ESAT-6 and $4.3(0.1-16.9)$ SFCs per 250000 PBMCs for CFP-10, and in the BAL the median for both ESAT-6 and CFP-10 was low with 0 SFCs per 250000 BALCs. Figure 2 and supplementary figure S1 show the number of SFCs in 250000 PBMCs and BALCs in patients with

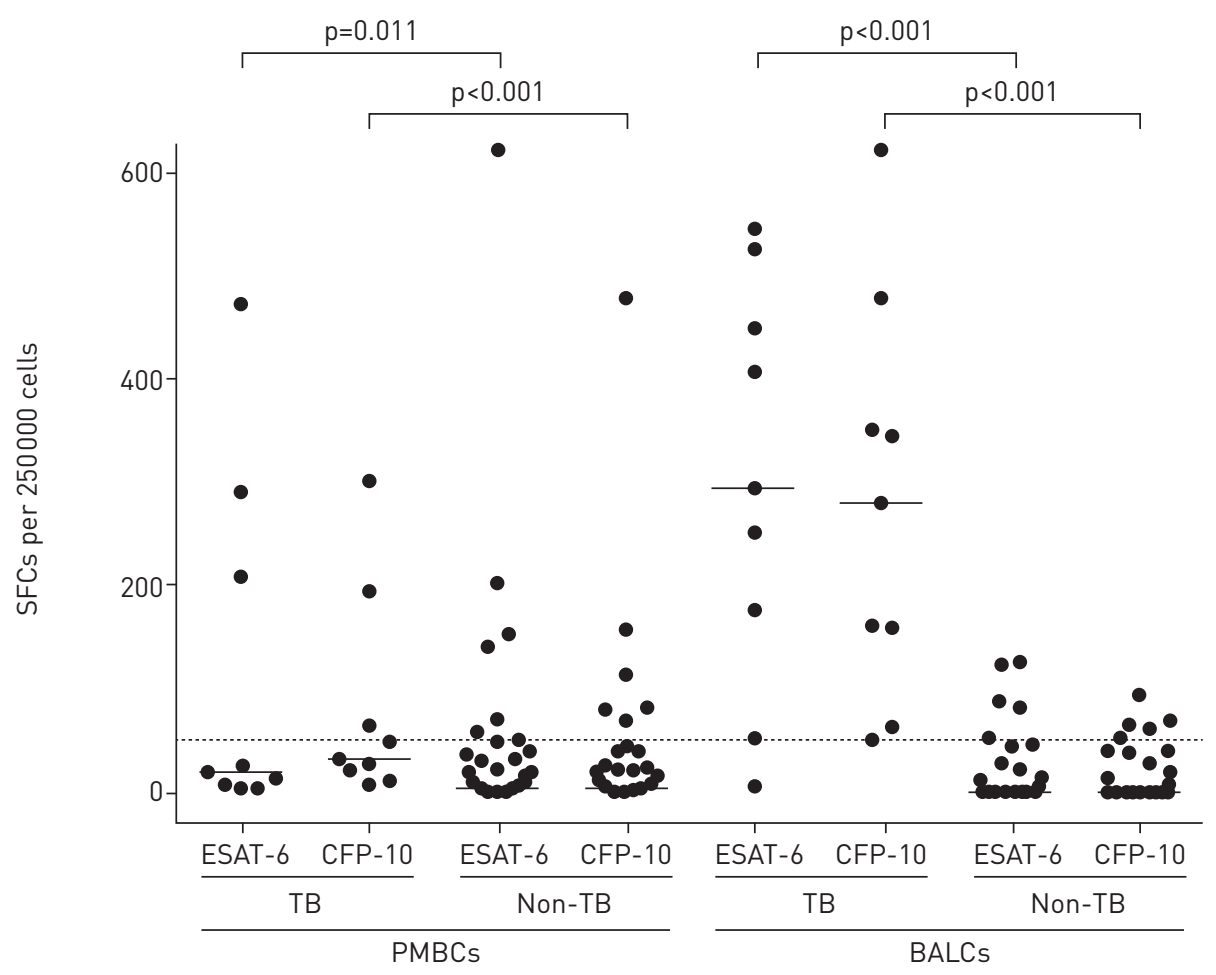

FIGURE 2 Number of spot-forming cells (SFCs) of early secretory antigenic target (ESAT)-6- and culture filtrate protein (CFP)-10-specific peripheral blood mononuclear cells (PBMCs) and bronchoalveolar lavage cells (BALCs) per 250000 cells in individuals where tuberculosis was excluded (non-TB) and in patients with culture-confirmed pulmonary TB with undetectable acid-fast bacilli on microscopy and negative GeneXpert results $(\mathrm{n}=9)$. A suggested cut-off $>50$ SFCs per 250000 cells is indicated. 
culture-confirmed pulmonary TB but undetectable AFB on microscopy and negative GeneXpert results compared with patients in whom TB had been excluded.

Concentration of antigen-specific lymphocytes at the site of infection

To account for the different percentages of lymphocytes between individuals, SFC results in PBMCs and BAL were normalised for 1000000 lymphocytes. In patients with culture-confirmed pulmonary $\mathrm{TB}$, the median (IQR) of antigen-specific lymphocytes per 1000000 in peripheral blood was 121 (43.9-1228.8) SFCs for ESAT-6 and 270 (109.8-1363.8) SFCs for CFP-10, while in the BAL the median (IQR) was 6370 (4595.2-14541.7) SFCs for ESAT-6 and 6598.5 (3857.7-13503.7) SFCs for CFP-10. By comparison, patients with a diagnosis other than TB had a median (IQR) of 25.5 (0.8-99.9) SFCs for ESAT-6- and 27 (0.7-93.8) SFCs for CFP-10-specific lymphocytes in peripheral blood, while in BAL they had a median (IQR) of 0 (0-0) SFCs for ESAT-6- and 0 (0-0) SFCs for CFP-10-specific lymphocytes per 1000000 lymphocytes. Figure 3 and supplementary figure S2 show the number of SFCs per 1000000 lymphocytes in PBMCs and BALCs in patients with culture-confirmed pulmonary TB but negative AFB microscopy/ negative GeneXpert results compared with patients without TB.

\section{Differentiation of active TB from non-TB}

To improve the discrimination between patients without TB and those with culture-confirmed pulmonary TB but undetectable AFB on microscopy and negative GeneXpert results in BAL, different cut-offs were investigated. A cut-off of $>50$ SFCs for ESAT-6- or CFP-10-specific cells from 250000 BALCs provides a sensitivity of $100 \%$ and a specificity of $91 \%$ (95\% CI $84-98 \%)$. Considering the proportion of lymphocytes, a cut-off of $>4000$ antigen-specific lymphocytes for either ESAT-6 or CFP-10 per 1000000 BAL lymphocytes had a sensitivity of $89 \%$ (95\% CI $68-100 \%)$ and a specificity of $97 \%$ (95\% CI $93-100 \%)$. To account for the different percentages of lymphocytes in BAL and peripheral blood, a recruitment factor was defined as SFCs in BALCs/SFCs in PBMCs multiplied by the lymphocyte factor (\% of lymphocytes in PBMCs/\% lymphocytes in BALCs). A recruitment factor of at least 6 for ESAT-6 or CFP-10 had a sensitivity of $67 \%$ (95\% CI 36-97\%) and a specificity of 83\% (95\% CI 74-92\%).

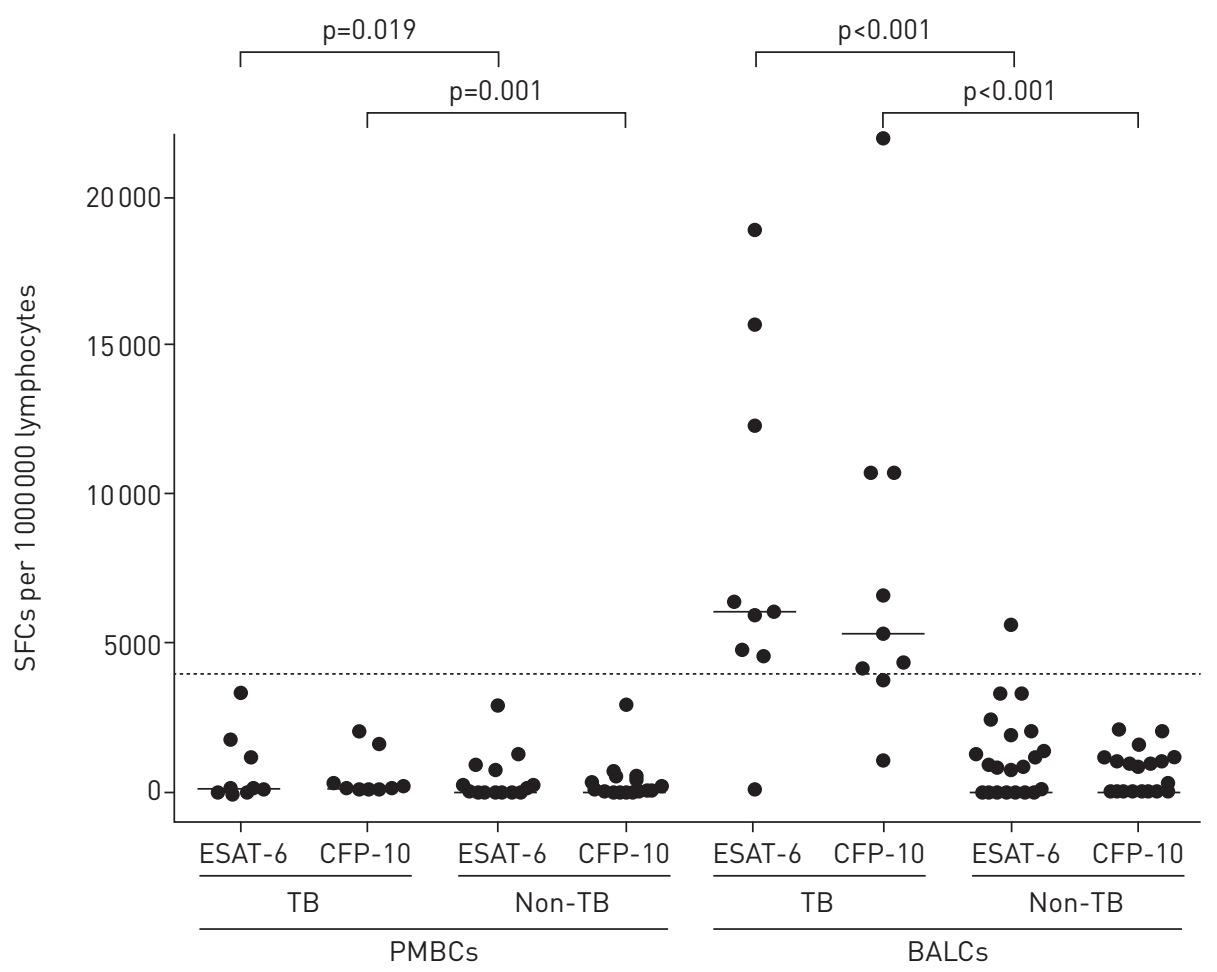

FIGURE 3 Number of spot-forming cells (SFCs) of early secretory antigenic target (ESAT)-6- and culture filtrate protein (CFP)-10-specific peripheral blood mononuclear cells (PBMCs) and bronchoalveolar lavage cells (BALCs) per 1000000 lymphocytes in individuals where tuberculosis was excluded (non-TB) and in patients with culture-confirmed pulmonary TB with undetectable acid-fast bacilli on microscopy and negative GeneXpert results (n=9). A suggested cut-off $>4000$ SFCs per 1000000 lymphocytes is indicated. 
TABLE 3 Performance of different cut-offs of culture filtrate protein (CFP)-10 or early secretory antigenic target (ESAT)-6 for Mycobacterium tuberculosis-specific bronchoalveolar lavage cell (BALC) ELISPOT for the diagnosis of active tuberculosis

\begin{tabular}{|c|c|c|c|c|c|c|c|c|c|c|}
\hline \multirow{2}{*}{$\begin{array}{l}\text { CFP-10 or ESAT-6 } \\
\text { cut-off }\end{array}$} & \multirow{2}{*}{$\begin{array}{c}\text { Sensitivity \% } \\
{[95 \% \mathrm{CI}]}\end{array}$} & \multirow{2}{*}{$\begin{array}{c}\text { Specificity \% } \\
{[95 \% \mathrm{CI}]}\end{array}$} & \multirow{2}{*}{$\begin{array}{l}\text { PPV \% } \\
(95 \% \mathrm{CI})\end{array}$} & \multirow{2}{*}{$\begin{array}{l}\text { NPV \% } \\
(95 \% \mathrm{CI})\end{array}$} & \multirow[t]{2}{*}{ CC \% } & \multirow{2}{*}{$\begin{array}{c}\text { LR+ \% } \\
{[95 \% \mathrm{CI}]}\end{array}$} & \multirow{2}{*}{$\begin{array}{l}\text { LR- \% } \\
(95 \% \mathrm{CI})\end{array}$} & \multirow{2}{*}{$\begin{array}{l}\text { False positive } \\
\text { (treated } \\
\text { unnecessarily) }\end{array}$} & \multicolumn{2}{|c|}{ AUC } \\
\hline & & & & & & & & & ESAT-6 & CFP-10 \\
\hline$>50$ BALCs & $\begin{array}{c}100 \\
(100-100)\end{array}$ & $\begin{array}{c}91 \\
(84-98)\end{array}$ & $\begin{array}{c}60 \\
(35-85)\end{array}$ & $\begin{array}{c}100 \\
(100-100)\end{array}$ & 91.9 & $\begin{array}{c}10.8 \\
(5.1-23.2)\end{array}$ & $0^{\#}$ & $6 / 100(6 \%)$ & 0.968 & 0.985 \\
\hline $\begin{array}{l}>4000 \mathrm{BAL} \\
\text { lymphocytes }\end{array}$ & $\begin{array}{c}89 \\
(68-100)\end{array}$ & $\begin{array}{c}97 \\
(93-100)\end{array}$ & $\begin{array}{c}80 \\
(55-100)\end{array}$ & $\begin{array}{c}98 \\
(95-100)\end{array}$ & 95.9 & $\begin{array}{c}28.9 \\
(7.2-115.3)\end{array}$ & $\begin{array}{c}0.1 \\
(0.02-0.7)\end{array}$ & $2 / 100(2 \%)$ & 0.973 & 0.978 \\
\hline $\begin{array}{c}\text { Recruitment } \\
\text { factor } \geqslant 6 \text { T }\end{array}$ & $\begin{array}{c}67 \\
(36-97)\end{array}$ & $\begin{array}{c}83 \\
(74-92)\end{array}$ & $\begin{array}{c}35 \\
(13-58)\end{array}$ & $\begin{array}{c}95 \\
(89-100)\end{array}$ & 80.8 & $\begin{array}{c}3.9 \\
(1.9-7.9)\end{array}$ & $\begin{array}{c}0.4 \\
(0.2-1)\end{array}$ & $11 / 100$ (11\%) & 0.879 & 0.910 \\
\hline
\end{tabular}

The test performance was computed for the 100 individuals who underwent bronchoscopy with BAL. PPV: positive predictive value; NPV: negative predictive value; CC: correctly classified; LR: likelihood ratio; AUC: area under the receiver operating characteristic curve. ${ }^{\#}: 95 \% \mathrm{Cl}^{\circ}$ cannot be calculated; ": spot-forming cells (SFCs) in BALCs/SFCs in PBMCs multiplied by the lymphocyte factor (\% of lymphocytes in PBMCs/ $\%$ lymphocytes in BALCs); ${ }^{+}$: SFCs in BALCs/SFCs in PBMCs.

The area under the ROC curve (AUC) to differentiate patients without TB from the subgroup of patients with culture-confirmed pulmonary TB but undetectable AFB on microscopy and negative GeneXpert results was 0.968 for ESAT-6- and 0.985 for CFP-10-specific BALCs, while the AUC for PBMCs was 0.760 for ESAT-6 and 0.841 for CFP-10. When evaluating the 1000000 lymphocytes from the BALCs only, the AUC was 0.973 for ESAT-6 and 0.978 for CFP-10.

Table 3 shows the performance of ELISPOT on BALCs using these different cut-offs. Supplementary figure S3 shows the graphs of the respective AUCs.

In summary, among 166 patients who were admitted with a presumptive diagnosis of pulmonary $\mathrm{TB}$, the diagnosis was confirmed by $M$. tuberculosis culture in 81 patients (48.8\%). 66 out of 81 (81.5\%) initially had a positive GeneXpert result from sputum and in addition six out of 81 (7.4\%) were diagnosed by BAL GeneXpert (together 72 out of $81(88.9 \%)$ ). In eight out of the nine out of $81(88.8 \%)$ patients with negative results on sputum and BAL by microscopy and GeneXpert, BAL ELISPOT identified patients with culture-confirmed TB correctly with a specificity of $97 \%$.

With a stepwise analysis of GeneXpert $(2 \mathrm{~h})$ and BAL ELISPOT only for patients with a negative BAL GeneXpert test result, the combined sensitivity and specificity for a rapid diagnosis of pulmonary TB was $98.8 \%$ and $97.6 \%$, respectively (table 4 ).

\section{Discussion}

We evaluated the accuracy of a stepwise approach consisting of an initial molecular test, i.e. GeneXpert, followed by a $M$. tuberculosis-specific BAL ELISPOT for the diagnosis of pulmonary TB. Using BAL GeneXpert and BAL ELISPOT sequentially, the diagnostic accuracy for sputum smear- and GeneXpert-negative pulmonary TB was $97 \%$. The diagnosis became available within the first days of presentation to the hospital.

TABLE 4 Performance of a combined approach of GeneXpert and bronchoalveolar lavage cell (BALC) ELISPOT for the diagnosis of active tuberculosis

\begin{tabular}{|c|c|c|c|c|c|c|c|c|}
\hline Cut-off & $\begin{array}{c}\text { Sensitivity \% } \\
\text { (95\% CI) }\end{array}$ & $\begin{array}{c}\text { Specificity \% } \\
(95 \% \mathrm{CI})\end{array}$ & $\begin{array}{l}\text { PPV \% } \\
(95 \% \mathrm{CI})\end{array}$ & $\begin{array}{l}\text { NPV \% } \\
(95 \% \mathrm{CI})\end{array}$ & CC \% & $\begin{array}{c}\text { LR+ \% } \\
(95 \% \mathrm{CI})\end{array}$ & $\begin{array}{l}\text { LR- \% } \\
(95 \% \mathrm{CI})\end{array}$ & $\begin{array}{l}\text { False positive } \\
\text { (treated } \\
\text { unnecessarily) }\end{array}$ \\
\hline
\end{tabular}

Test performance of this stepwise approach of combined GeneXpert and ELISPOT refers to 166 individuals (100 BAL and 66 sputum). PPV: positive predictive value; NPV: negative predictive value; CC: correctly classified; LR: likelihood ratio. 
GeneXpert has greatly improved the diagnosis of TB. If GeneXpert is performed on two different sputum samples, $>90 \%$ of adult patients with pulmonary TB are correctly identified by GeneXpert within the first days of presentation to a healthcare facility [25]. However, the sensitivity of GeneXpert is lower in sputum smear-negative and in extrapulmonary TB $[25,26]$. If results from GeneXpert tests are negative, patients could remain undiagnosed for several weeks until results of $M$. tuberculosis cultures become available.

M. tuberculosis-specific ELISPOT performed on cells from BAL is able to close this diagnostic gap. In the past decade several studies have shown that M. tuberculosis-specific ELISPOT performed on specimens from the site of the disease, e.g. BAL in pulmonary $\mathrm{TB}$, pleural or pericardial effusion in $\mathrm{TB}$ pleurisy or TB pericarditis, respectively, ascites in TB peritonitis, or cerebrospinal fluid in TB meningitis [27-30], can improve the case detection rate of TB in paucibacillary disease, but the added value of a stepwise approach of GeneXpert and ELISPOT had not been investigated so far.

In active TB, M. tuberculosis-specific lymphocytes are recruited from the blood into the disease-affected organ [31,32]. Active disease is more likely with increasing concentration of $M$. tuberculosis-specific cells at the site of infection compared with peripheral blood [24]. Hofland et al. [33] suggested a ratio of SFCs in BAL/SFCs in blood with a cut-off $>1$. However, one needs to consider that lymphocytes predominate in peripheral blood, whereas the proportion in BAL is considerably lower [34]. These differences in percentages of lymphocytes in PBMCs and BALCs should be acknowledged individually by calculating a lymphocyte-dependent recruitment factor of ESAT-6- and CFP 10-specific T-cells between blood and BALCs or by normalising the SFCs per 250000 cells to 1000000 lymphocytes $[11,24]$.

Optimal result were obtained in this study with a cut-off of $>4000$ lymphocytes per 1000000 lymphocytes in BAL. When applying this cut-off, patients with paucibacillary pulmonary TB could be identified with a sensitivity of $89 \%$ and a specificity of $97 \%$. This cut-off provided an excellent negative predictive value of $98 \%$ and a high positive likelihood ratio of 28.9 .

As with most studies from low TB incidence countries, our study is limited by the sample size of patients with culture-confirmed active TB and negative GeneXpert results from sputum and BAL. Performing BAL ELISPOT and flow cytometry is also substantially more labour intensive and requires considerably more technician time than automated $M$. tuberculosis-specific nucleic acid amplification by GeneXpert. However, most patients who are suspected of having pulmonary TB in low TB incidence countries with sufficient economical resources will undergo bronchoscopy when results of sputum smear microscopy and sputum GeneXpert analysis are negative, and also to exclude alternative diseases such as sarcoidosis, cryptogenic organising pneumonia, lung cancer and others. Furthermore, the results cannot be generalised to high TB burden countries, where repeated exposure to $M$. tuberculosis may alter BAL ELISPOT responses [14] and performance of ELISPOT would be challenging due to limited laboratory capacity.

Due to the high sensitivity for pulmonary TB of GeneXpert performed on respiratory specimens, the performance of $M$. tuberculosis-specific BAL ELISPOT can be limited to patients where the GeneXpert results from sputum and BAL are negative and when the differential diagnosis of active pulmonary TB is still to be considered.

In conclusion, in low $\mathrm{TB}$ incidence counties, nearly all patients with active pulmonary $\mathrm{TB}$ can be identified within the first few days of clinical presentation by using a stepwise testing strategy with GeneXpert and BAL ELISPOT.

Acknowledgements: The authors thank the staff at the National Reference Center for Mycobacteria (Borstel, Germany), especially Birgit Voss and Kerstin Klein for their administrative support with mycobacterial culture results, and Cordula Ehlers and Dagmar Schaub (Clinical Infectious Diseases, Borstel, Germany) for databanking.

Conflict of interest: J. Heyckendorf has received personal fees for sponsor-independent lectures at sponsored symposia from Chiesi, Gilead, Janssen, Lucane and Hain, outside the submitted work. C. Lange has received personal fees for sponsor-independent lectures at sponsored symposia from Chiesi, Gilead, Abbvie, MSD, Becton Dickinson, Janssen, Lucane, Novartis and Thermo Fisher, outside the submitted work. B. Kalsdorf has received personal fees for sponsor-independent lectures at sponsored symposia from Oxford Immunotec and Lucane, outside the submitted work.

Support statement: C. Lange and J. Heyckendorf are supported by the German Center for Infection Research (DZIF). Funding information for this article has been deposited with the Crossref Funder Registry.

\section{References}

World Health Organization. Global Tuberculosis Report 2017. Geneva, WHO, 2017.

2 Badak FZ, Kiska DL, Setterquist S, et al. Comparison of mycobacteria growth indicator tube with BACTEC 460 for detection and recovery of mycobacteria from clinical specimens. J Clin Microbiol 1996; 34: 2236-2239.

3 Pheiffer C, Carroll NM, Beyers N, et al. Time to detection of Mycobacterium tuberculosis in BACTEC systems as a viable alternative to colony counting. Int J Tuberc Lung Dis 2008; 12: 792-798. 
4 Robert Koch Institute. Bericht zur Epidemiologie der Tuberkulose in Deutschland für 2016. [Report on the Epidemiology of Tuberculosis in Germany for 2016.] Berlin, Robert Koch Institute, 2017.

5 Steingart KR, Schiller I, Horne DJ, et al. Xpert MTB/RIF assay for pulmonary tuberculosis and rifampicin resistance in adults. Cochrane Database Syst Rev 2014; 1: CD009593.

6 De Jong R, Brouwer M, Hooibrink B, et al. The CD27- subset of peripheral blood memory CD4 $4^{+}$lymphocytes contains functionally differentiated $\mathrm{T}$ lymphocytes that develop by persistent antigenic stimulation in vivo. Eur $\mathrm{J}$ Immunol 1992; 22: 993-999.

7 Silver RF, Zukowski L, Kotake S, et al. Recruitment of antigen-specific Th1-like responses to the human lung following bronchoscopic segmental challenge with purified protein derivative of Mycobacterium tuberculosis. Am J Respir Cell Mol Biol 2003; 29: 117-123.

8 Nikitina IY, Kondratuk NA, Kosmiadi GA, et al. Mtb-specific CD27 ${ }^{\text {low }}$ CD4T cells as markers of lung tissue destruction during pulmonary tuberculosis in humans. PLoS One 2012; 7: e43733.

9 Nemeth J, Rumetshofer R, Winkler H-M, et al. Active tuberculosis is characterized by an antigen-specific and strictly localized expansion of effector T cells at the site of infection. Eur J Immunol 2012; 42: 2844-2850.

10 Kapina MA, Shepelkova GS, Mischenko VV, et al. CD27 ${ }^{\text {low }}$ CD4T lymphocytes that accumulate in the mouse lungs during mycobacterial infection differentiate from $\mathrm{CD} 27^{\text {high }}$ precursors in situ, produce IFN-gamma, and protect the host against tuberculosis infection. J Immunol 2007; 178: 976-985.

11 Jafari C, Ernst M, Kalsdorf B, et al. Rapid diagnosis of smear-negative tuberculosis by bronchoalveolar lavage enzyme-linked immunospot. Am J Respir Crit Care Med 2006; 174: 1048-1054.

12 Jafari C, Thijsen S, Sotgiu G, et al. Bronchoalveolar lavage enzyme-linked immunospot for a rapid diagnosis of tuberculosis: a Tuberculosis Network European Trials group study. Am J Respir Crit Care Med 2009; 180: 666-673.

13 Cattamanchi A, Ssewenyana I, Nabatanzi R, et al. Bronchoalveolar lavage enzyme-linked immunospot for diagnosis of smear-negative tuberculosis in HIV-infected patients. PLoS One 2012; 7: e39838.

14 Dheda K, van Zyl-Smit RN, Meldau R, et al. Quantitative lung $\mathrm{T}$ cell responses aid the rapid diagnosis of pulmonary tuberculosis. Thorax 2009; 64: 847-853.

$15 \mathrm{Li} \mathrm{H}$, Yang L, Zheng CY, et al. Use of bronchoalveolar lavage enzyme-linked immunospot for diagnosis of smear-negative pulmonary tuberculosis. Int J Tuberc Lung Dis 2012; 16: 1668-1673.

16 Losi M, Bossink A, Codecasa L, et al. Use of a T-cell interferon-gamma release assay for the diagnosis of tuberculous pleurisy. Eur Respir J 2007; 30: 1173-1179.

17 Kim MC, Kim SM, Lee SO, et al. A diagnostic algorithm for tuberculous pleurisy using the ELISpot assay on peripheral blood and pleural effusion. Infect Dis 2016; 48: 688-694.

18 Bian S, Zhang Y, Zhang L, et al. Diagnostic value of interferon- $\gamma$ release assays on pericardial effusion for diagnosis of tuberculous pericarditis. PLoS One 2016; 11: e0165008.

19 Lu D, Chen C, Yu S, et al. Diagnosis of tuberculous meningitis using a combination of peripheral blood T-SPOT. TB and cerebrospinal fluid interferon- $\gamma$ detection methods. Lab Med 2016; 47: 6-12.

20 Lei Y, Yi FM, Zhao J, et al. Utility of in vitro interferon- $\gamma$ release assay in differential diagnosis between intestinal tuberculosis and Crohn's disease. J Dig Dis 2013; 14: 68-75.

21 Häussinger K, Ballin A, Becker HD, et al. Recommendations for quality standards in bronchoscopy. Pneumologie 2004; 58: 344-356.

22 Bossuyt PM, Reitsma JB, Bruns DE, et al. STARD 2015: an updated list of essential items for reporting diagnostic accuracy studies. BMJ 2015; 351: h5527.

23 World Health Organization. Xpert MTB/RIF Implementation Manual. Technical and Operational 'How-To': Practical Considerations. Geneva, WHO, 2014.

24 Jafari C, Ernst M, Strassburg A, et al. Local immunodiagnosis of pulmonary tuberculosis by enzyme-linked immunospot. Eur Respir J 2008; 31: 261-265.

25 Boehme CC, Nabeta P, Hillemann D, et al. Rapid molecular detection of tuberculosis and rifampin resistance. N Engl J Med 2010; 363: 1005-1015.

26 Boehme CC, Nicol MP, Nabeta P, et al. Feasibility, diagnostic accuracy, and effectiveness of decentralised use of the Xpert MTB/RIF test for diagnosis of tuberculosis and multidrug resistance: a multicentre implementation study. Lancet 2011; 377: 1495-1505.

27 Pang $\mathrm{C}$, Wu Y, Wan C, et al. Accuracy of the bronchoalveolar lavage enzyme-linked immunospot assay for the diagnosis of pulmonary tuberculosis: a meta-analysis. Medicine 2016; 95: e3183.

28 Li ZZ, Qin WZ, Li L, et al. Accuracy of enzyme-linked immunospot assay for diagnosis of pleural tuberculosis: a meta-analysis. Genet Mol Res 2015; 14: 11672-11680.

29 Tebruegge M, Ritz N, Koetz K, et al. Availability and use of molecular microbiological and immunological tests for the diagnosis of tuberculosis in Europe. PLoS One 2014; 9: e99129.

30 Jafari C, Ernst M, Kalsdorf B, et al. Comparison of molecular and immunological methods for the rapid diagnosis of smear-negative tuberculosis. Int J Tuberc Lung Dis 2013; 17: 1459-1465.

31 Barnes PF, Lu S, Abrams JS, et al. Cytokine production at the site of disease in human tuberculosis. Infect Immun 1993; 61: 3482-3489.

32 Flynn JL, Chan J. Immunology of tuberculosis. Annu Rev Immunol 2001; 19: 93-129.

33 Hofland RW, Thijsen S, van Lindert AS, et al. Positive predictive value of ELISpot in BAL and pleural fluid from patients with suspected pulmonary tuberculosis. Infect Dis 2017; 49: 347-355.

34 Costabel U. Atlas of Bronchoalveolar Lavage. London, Chapman \& Hall Medical, 1999. 